\title{
The Impact of Benson's Relaxation Technique on the Quality of Sleep in the Elderly
}

\author{
Mitra Habibollahpour, MSc; Fatemeh Ranjkesh, MSc; Seyedeh Ameneh Motalebi, PhD;
} Fatemeh Mohammadi, PhD

\begin{abstract}
Objective: This study examined the effect of Benson's relaxation technique in the quality of sleep of the elderly. Method: The study employed a randomized clinical trial design. A total of 75 elderly suffering from sleep disorder were randomly assigned to either the intervention $(n=38)$ or control $(n=37)$ group. The intervention group received Benson's relaxation technique twice a day for 20 minutes through 4 weeks. Petersburg Sleep Quality Index was used to collect the data. Independent and paired $t$ tests were employed to analyze the data.

Findings: The results of the paired $t$ tests showed significant improvements in intervention group on quality of sleep and its 5 subscales including subjective sleep quality, sleep latency, sleep duration, and sleep efficiency, and the daytime dysfunction improved significantly ( $P \quad .000$ ). In addition, independent $t$ tests showed significant differences between the intervention and control groups in the quality of sleep and its 4 subscales including the subjective sleep quality, sleep latency, sleep duration, and habitual sleep efficiency after implementation of intervention ( $P$.000).

Conclusion: The results of the present study may support the efficacy of this simple, available, and cost-effective technique on the quality of sleep of elderly people.

Key words: Benson's relaxation technique, elderly, quality of sleep
\end{abstract}

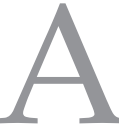
ging is a gradual and continuous process of natural change in all the beings, during which the capacity for cell division, growth, and performance decreases, and finally it leads to death. ${ }^{1}$ One of the challenges for the elderly, in particular, is living with various health problems. ${ }^{2}$ Of the common problems in aging is sleep disorder that affects the quality of life of the elderly. ${ }^{3}$ Sleep is considered as a basic human need and is included in the Maslow's needs gradation in physiological needs and

Author Affiliation: Nursing and Midwifery Faculty, Qazvin University of Medical Sciences, Qazvin, Iran.

The authors thank all of the elderly people who participated in this study. The authors have disclosed that they have no significant relationships with, or financial interest in, any commercial companies pertaining to this article.

Correspondence: Fatemeh Mohammadi, PhD, Nursing and Midwifery Faculty, Qazvin University of Medical Sciences, Shahid Bahonar Blvd, 59811-34197, Qazvin, Iran (fmohammadi@qums.ac.ir). provides the human's body with an opportunity to reenergize and get rid of tension. ${ }^{4}$ Researches showed that aging is associated with a decrease in the quality and quantity of sleep ${ }^{5,6}$ because age-related changes lead to a lighter sleep and decrease in the sleep efficiency. ${ }^{7}$ According to Wu et al, ${ }^{8}$ $49 \%$ of the elderly residing in China had a weak quality of sleep. ${ }^{8}$ In Iran, $67 \%$ of the elderly have sleep disorders, and $61 \%$ of these people suffer from insomnia. ${ }^{9}$ The most common sleep complaints in the elderly include difficulty falling asleep, waking up during the night, waking too early, and daily drowsiness. ${ }^{10}$

The real amount of sleep decreases as the age goes up. ${ }^{11}$ In the elderly persons, the delta phase (deep sleep stage) duration decreases, as they spend about $10 \%$ of their night sleep in this phase. So, in ageing, sleep is light, and the elderly wake up frequently during the night. Common physical conditions of the ageing also disturb the quality of sleep. For instance, many elderly people suffer from sleep disorders due to the night pain resulting from arthritis, or using diuretic drugs to control high blood pressure causes frequent night wakes to urinate. ${ }^{12}$

The desirable sleep increases the physical health, decreases the dullness and anxiety, and strengthens the ability to adapt and concentrate. ${ }^{13}$ On the contrary, lack of sleep brings a sense of tiredness, headache, concentration deficit disorder, day narcolepsy, weak perception, susceptibility to infection, and even cancer and cardiovascular diseases. ${ }^{13,14}$ Helbig et a ${ }^{15}$ showed a high relationship between low quality of sleep and the risk of falling among the elderly older than 75 years. ${ }^{15}$ Among the reasons of low sleep quality in the elderly are the external factors such as lighting, noise, environment temperature, and nursing care, and internal factors such as pain, illness, drugs, and anxiety and its related psychological changes. ${ }^{16,17}$

Irrespective of suffering from different chronic conditions, the crisis of losing the spouse or friends, loneliness, retirement, a decrease in the functional abilities, appearance changes, fall income, decrease of social security, and negative social attitudes affect the elderly's quality of sleep. ${ }^{18}$

There are various methods to deal with sleep disorders. ${ }^{19}$ The elderly usually use soporific drugs in such a way that $39 \%$ of these drugs are used by the elderly older than 60 years. ${ }^{12,20}$ However, these drugs alleviate sleep disorders temporarily and most of them cause drug 